08

\title{
Температура поверхности конденсации как инструмент для исследования механизмов формирования пленок
}

\author{
(С) Л.Р. Шагинян, С.А. Фирстов, И.Ф. Копылов \\ Институт проблем материаловедения НАН Украины, \\ 03142 Киев, Украина \\ e-mail: Irshag49@gmail.com
}

(Поступило в Редакцию 11 июля 2017 г.)

\begin{abstract}
Повышение температуры поверхности конденсации в процессе роста пленки есть результат диссипации различных видов энергии на этой поверхности. Одним из видов энергии является теплота химических реакций, которая выделяется в процессе осаждения пленки, получаемой реакционным магнетронным распылением. Мониторинг температуры поверхности, $T_{\text {surf }}$, при осаждении пленок TiN при реакционном $\left(\mathrm{Ti}-\mathrm{in}-\mathrm{N}_{2}\right)$ и безреакционном ( $\mathrm{TiN}-\mathrm{in}-\mathrm{Ar}$ и $\left.\mathrm{TiN}-\mathrm{in}-\mathrm{N}_{2}\right)$ методах распыления показал, что максимальная величина этой температуры наблюдалась при реакционном методе, а в ряду безреакционных модификаций ( TiN-in-Ar) - $\left(\mathrm{TiN}-\mathrm{in}-\mathrm{N}_{2}\right)$ она убывала. В то же время состав и кристаллическая структура пленок $\mathrm{TiN}$ не зависели от метода получения и соответствовали таковым у объемного нитрида титана. На основании этих результатов предложен механизм формирования пленок, получаемых указанными методами. Предполагается, что в процессе реакционного распыления пленка формируется на поверхности конденсации путем реакции между атомами азота и титана, тогда как в безреакционных методах распыления формирование пленки осуществляется из молекул TiN.
\end{abstract}

DOI: 10.21883/JTF.2018.04.45727.2428

\section{Введение}

Все свойства пленки целиком определяются ее двумя фундаментальными характеристиками - составом и структурой. В свою очередь, последние являются функциями двух особенностей пленкообразующих частиц (ППЧ), поступающих на поверхность конденсации, их энергией и составом. Существует большой объем информации, собранной в монографиях, отдельных оригинальных работах и обзорах, в которой выявлена важная роль энергии частиц, бомбардирующих растущую пленку, в формировании ее разнообразных свойств, включая состав, кристаллическую и микроструктуру [1-5]. В то же время работ, в которых исследовано влияние типа пленкообразующих частиц (атомы, молекулы, атомные кластеры) на состав и структуру пленок, чрезвычайно мало. Однако даже на интуитивном уровне ясно, что размер ППЧ должен играть заметную роль в формировании структуры пленки. Действительно, подвижность ППЧ на поверхности конденсации существенно зависит от их размера и типа. Подвижность более крупных частиц на поверхности конденсации меньше, чем у атомов, более того, их присутствие ограничивает подвижность последних благодаря столкновениям с более крупными и массивными молекулами и кластерами. При этом, чем выше подвижность атомов, тем более совершенной оказывается структура пленки [6]. Например, при исследовании механизмов формирования пленок (W,Ti)N, осаждаемых реакционным магнетронным распылением, было показано, что присутствие молекул WN и $\mathrm{TiN}$ на поверхности конденсации играет ключевую роль в формировании кристаллической и микроструктуры пленки. Пленка, осажденая из смешанного потока, содержащего атомы и молекулы, формируется как бифазный нанокристаллический материал $[\beta-\mathrm{W}+(\mathrm{W}, \mathrm{Ti}) \mathrm{N}]$ с особо малым размером зерна. Пленка же, осажденная преимущественно из потоков атомов металлов и азота, сформирована монофазным материалом (W,Ti) ${ }_{2} \mathrm{~N}$ с размером зерна в 2-4 раза большим [7].

Очевидно, что исследование влияния типа ППЧ на свойства пленки более сложная задача, чем исследование эффектов ионной бомбардировки. Прямое исследование состава ППЧ, особенно если они формируются в диапазоне относительно высоких давлений, в которых функционируют магнетронные системы распыления ( 10 $0^{-2}-10^{-3}$ Torr), связано со сложным и дорогостоящим экспериментальным оборудованием, таким, как квадрупольная масс-спектрометрия [7], лазерная фотоионизация распыленных (испаренных) частиц с последующей обработкой спектров [8] и др.

В этой связи создание новых подходов и методов, позволяющих исследовать состав и тип ППЧ, поступающих на поверхность конденсации, и выявление их роли в формировании свойств пленки, является перспективным и важным вызовом как в конкретной области физики пленок, так и для решения более общей проблемы формирования твердого тела из паровой фазы.

Настоящяя работа посвящена исследованию механизма формирования пленок нитрида титана, получаемых различными модификациями метода магнетронного распыления, с помощью нового подхода. Подход основан на простом и точном калориметрическом методе измерения температуры поверхности конденсации, повышение которой наблюдается в процессе роста пленки [9]. 
Используя результаты измерения температуры поверхности и исследования состава и кристаллической структуры пленок, мы предлагаем качественный механизм формирования пленок нитрида титана, получаемых реакционным и безреакционным магнетронным распылением мишеней из Ti и TiN.

\section{1. Техника эксперимента}

\section{1. Методика получения пленок}

Для решения указанных задач нами были использованы реакционная и безреакционная модификации магнетронного распыления на постоянном токе мишени из металлического титана и керамической мишени из нитрида титана. Мишени представляли собой диски диаметром $55 \mathrm{~nm}$ и толщиной $4 \mathrm{~nm}$. Мишень из нитрида титана изготовлена методом горячего прессования порошка TiN (97\% чистоты), тогда как мишень титана была получена токарным способом из слитка чистотой 99.99.

До проведения измерений камера откачивалась до давления $(1-2) \cdot 10^{-5}$ Torr, тогда как давление рабочих газов (аргона или азота) в камере в процессе исследований поддерживалось в пределах $(3-5) \cdot 10^{-3}$ Torr. Чистота использованных газов была не ниже 99.99. Для создания стабильных условий процесса мишень распыляли в течение $10 \mathrm{~min}$ при закрытой заслонке. Расстояние мишень-подложка во всех экспериментах составляло $50 \mathrm{~mm}$.

Такие параметры (давление рабочего газа и расстояние между мишенью и подложкой) обеспечивали движение распыленных частиц практически в баллистическом (бесстолкновительном) режиме.

Измерение температуры проводили при различных токах разряда на мишень, $I_{d}$, который варьровали в пределах $0.2-0.5 \mathrm{~A}$. Соответствующие напряжения на мишени изменялись в пределах $-350-500 \mathrm{~V}$. Для проведения этих измерений изготавливали соответствующие подложки, описание которых приведено ниже. Чтобы избежать путаницы в названиях отметим, что для температурных измерений подложку будем называть „образец“, тогда как во всех прочих исследованиях она будет называться подложкой.

Для анализа состава и кристаллической структуры пленки осаждали на подложки из полированных пластин монокристаллов кремния, а также свежих сколов монокристаллов $\mathrm{NaCl}$. Подложки из кремния перед осаждением очищали в ультразвуковой ванне в смеси ацетона и спирта $(50: 50)$ с последующей промывкой в дистиллированной воде и просушкой горячим воздухом.

Типичная толщина пленок для исследования состава составляла $1.5-5.0 \mu \mathrm{m}$, тогда как для исследования их структуры с помощью электронной микроскопии и электронографии она была $\leq 100 \mathrm{~nm}$. Скорость роста пленки рассчитывали как отношение толщины пленки ко времени ее осаждения, которая в зависимости от условий осаждения составляла $\sim 0.5-6 \AA / \mathrm{s}$. Величина скорости роста использовалась для расчета потока энергии, поступающего на поверхность конденсации в процессе формирования пленки.

\section{2. Методики исследования свойств пленок}

Кристаллическую структуру пленок исследовали с помощью электронографа ЭМР-100 при ускоряющем напряжении $75 \mathrm{kV}$ в режимах „на просвет“ и „на отражение“".

Точность измерения межплоскостных расстояний составляла $\Delta \approx \pm 0.01 \AA$ и $\Delta \approx \pm 0.03 \AA$ в режимах „на просвет“ и „на отражение“ соответственно. Для исследования структуры пленок методом „на просвет“ их осаждали на свежие сколы кристаллов $\mathrm{NaCl}$ с последующим отделением от подложки путем погружения в дистиллированную воду и помещением свободной пленки на медную сеточку для электронной микроскопии.

Химический состав пленок, осажденных на подложки из кремния, и керамической мишени из TiN исследовали методом спектрометрии энергии потерь электронов на приборе JSM-6U90LV. Чтобы снизить ошибку в измерении отношения N/Ti состав пленки исследовали в 3-5 точках. Эта мера позволяла определять отношение N/Ti с точностью не хуже $\sim 0.1 \%$, тогда как ошибка в определении содержания примесей (углерод и кислород) была $\sim 0.5 \%$. Толщину пленки измеряли двумя методами - прямым измерением толщины на поперечном сечении пленки с подложкой с помощью сканирующей электронной микроскопии и оптической микроинтерферометрией (микроинтерферометр Линника). Ошибка при измерении последним методом зависела от толщины пленки и в нашем случае (пленки были толщиной $1.5-5 \mu \mathrm{m})$ не превышала 5\% толщины.

\section{3. Методика измерения температуры поверхности}

\subsection{1. Принцип калориметрического метода измерения температуры поверхности}

Определение температуры поверхности конденсации, $G_{\text {surf }}$, заметно возрастающей при конденсации на ней пленки, основано на калориметрическом методе [10]. С этой целью in situ измеряется температура образца, $T_{\text {samp, }}$, изменяющаяся со временем $t$ в процессе роста пленки, и строится зависимость $T_{\mathrm{samp}}=f(t)$. Типичная $T_{\mathrm{samp}}=f(t)$ зависимость приведена на рис. $1, a$.

Схематически идея метода определения $T_{\text {surf }}$ представлена на рис. $1, b, c$. Температура образца, подвергаемого облучению любого типа, возрастает. Пока его температура столь низка, что потоком тепла, излучаемым образцом, можно пренебречь, величина потока энергии, поступающая на его поверхность, линейно возрастает и 


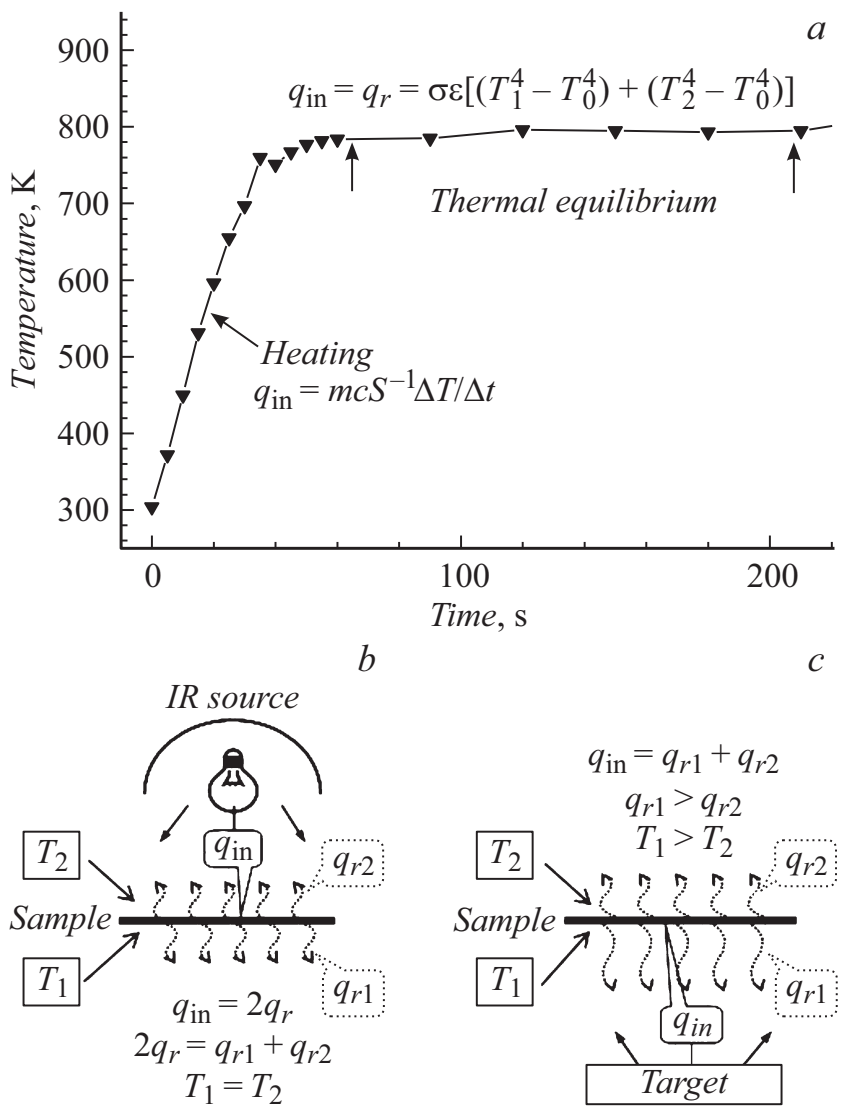

Рис. 1. $a-$ типичная кривая зависимости температуры от времени, $T=f(t)$, как основа для калориметрического метода измерения температуры. Уравнение (1) описывает восходящую линейную часть кривой, тогда как уравнение (4) описывает также линейную, но горизонтальную часть этой кривой, см. текст. Основываясь на этой кривой, можно рассчитать температуру поверхности, $T_{\text {surf, }}$ и излучательную способность, $\varepsilon$; $b$ - потоки тепла, $q_{r}$, излучаемые каждой поверхностью образца, равны; $c$ - потоки тепла, $q_{r}$, излучаемые каждой поверхностью, не равны.

может быть описана уравнением (1):

$$
q_{\mathrm{in}}=m c S^{-1} \frac{\Delta T}{\Delta t}
$$

где $m, c, S, \Delta T / \Delta t$ - масса, удельная теплоемкость, площадь образца и скорость роста температуры соответственно (восходящая часть кривой $T_{\text {samp }}=f(t)$ на рис. $1, a)$. Пусть образец представляет собой диск такой малой толщины, что излучением тепла с его образующей можно пренебречь. Если такой образец подвергается облучению ИК излучения, то потоки тепла, излучаемые обеими его сторонами, $q_{r}$, равны, и общий поток излучения образцом можно описать уравнением Стефана-Больцмана (2):

$$
q_{r}=2 \sigma \varepsilon\left(T_{\text {samp }}^{4}-T_{0}^{4}\right) .
$$

В уравнении (2) множитель „2“ указывает на равенство потоков $q_{r}, \sigma-$ постоянная Больцмана, $\varepsilon-$ излу- чательная способность, $T_{\text {samp }}$ измеряемая температура образца, $T_{0}$ - температура окружающей среды.

Когда температура образца достигнет максимума и перестанет изменяться, это будет означать равенство потоков энергии, поступающей на образец, и излучаемой им (уравнение (3)):

$$
q_{\text {in }}=q_{r},
$$

при условии, что тепло, излучаемое образцом, не отводится от него никаким другим механизмом.

На рис. 1, $a$ этот процесс изображен горизонтальным участком кривой $T_{\text {samp }}=f(t)$.

Если по какой-либо причине потоки тепла, излучаемые разными сторонами образца, оказываются не равными (т. е., $q_{r 1}>q_{r 2}$ ), это будет означать, что температура стороны, излучающей больший поток тепла, будет выше, чем противоположной (рис. 1,c). В этом случае

$$
q_{r 0}=q_{r 1}+q_{r 2}=\sigma \varepsilon\left[\left(T_{1}^{4}-T_{0}^{4}\right)+\left(T_{2}^{4}-T_{0}^{4}\right)\right],
$$

где $q_{r 0}=q_{r 1}+q_{r 2}-$ суммарный поток тепла от образца, $q_{r 1}, q_{r 2}$ потоки и $T_{1}, T_{2}$ - температуры каждой стороны образца соответственно, см. горизонтальную часть кривой на рис. $1, a$. Измеряя температуру $T_{1}$ термопарой, прикрепленной к образцу, рассчитывая по уравнению (1) $T_{\mathrm{samp}}=f(t)$ поток тепла, $q_{\text {in }}-$ поступающий на образец, находим температуру $T_{2}$ противоположной стороны образца:

$$
T_{2}=\sqrt[4]{\frac{q_{\text {in }}}{\sigma \varepsilon}-T_{1}^{4}+2 T_{0}^{4}} .
$$

Следует подчеркнуть, что для выполнения уравнений (4) и (5) излучательная способность обеих сторон образца должна быть одинакова.

\subsection{2. Экспериментальная реализация метода}

Приведенные рассуждения были применены для определения температуры поверхности образца с напыляемой стороны. В качестве образца был использован медный диск $40 \mathrm{~mm}$ в диаметре, толщиной $0.15 \mathrm{~mm}$, массой $m=3.45 \mathrm{~g}$, обе стороны которого в зависимости от проводимых измерений были покрыты слоем TiN или Ті толщиной $1.5-2 \mu \mathrm{m}$. Температуру образца $T_{\text {samp }}$ измеряли при помощи хромель-алюмелевой термопары (толщина каждой проволоки $-0.05 \mathrm{~mm})$, спай которой был механически прочно закреплен в образце. Образец был подвешен параллельно распыляемой мишени на расстоянии $50 \mathrm{~mm}$, как показано на рис. 2. Чтобы осуществить подвес образца, на диаметрально противоположных сторонах диска при его изготовлении были предусмотрены две полоски шириной по 2 и длиной по $3 \mathrm{~mm}$. Загибая полоску с одной стороны, с еe помощью механически прочно фиксировали спай термопары, а другой полоской - хромелевую проволоку, теплоизолированную от диска керамической соломкой (рис. $2, b)$. Натягивая с одной стороны термопару, а с 


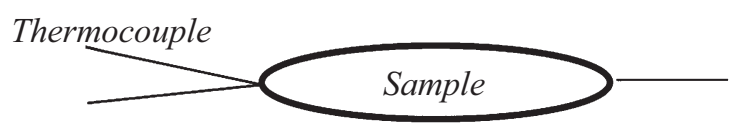

Shutter 2
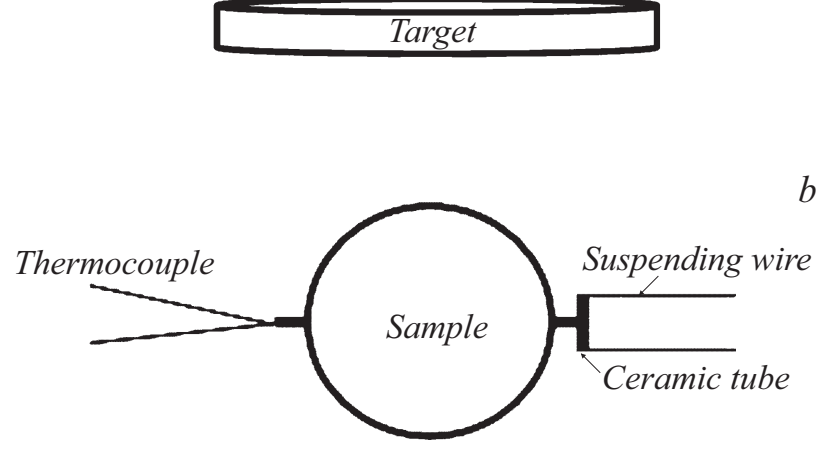

Рис. 2. Схема эксперимента для измерения температуры поверхности, $T_{\text {surf }}: a-$ вид сбоку, $b-$ вид сверху.

другой проволоку, диск подвешивали над мишенью, как показано на рис. 2, $a$.

Для расчета $T_{\text {surf }}$, ввиду несравнимо малой массы покрытия на образце его удельная теплоемкость была принята равной теплоемкости меди, $c=0.386 \mathrm{~J} \cdot(\mathrm{g} \cdot \mathrm{K})^{-1}$.

\subsection{3. Метод определения излучательной способности образца}

Для точного определения температуры поверхности, $T_{\text {surf, }}$ необходимо знать точное значение излучательной способности $\varepsilon$ образца. Для ее определения была разработана следующая методика. Медный диск (образец) закрепляли в камере, как показано на рис. 2, затем каждую его сторону распылением мишени покрывали соответствующим (TiN или Ti) слоем толщиной $2-2.5 \mu \mathrm{m}$. После чего образец, охлажденный до комнатной температуры, без развакуумирования камеры облучали лампой накаливания со стабилизированной мощностью $(300 \mathrm{~W})$, как показано на схеме рис. $1, b$. В процессе нагрева фиксировались данные изменения температуры образца $T_{\text {samp }}$ во времени, а по достижении теплового равновесия измерение продолжали еще в течение $10 \mathrm{~min}$. На основании полученных данных строилась зависимость $T_{\text {samp }}=f(t)$, типичный вид которой приведен на рис. $1, a$. Используя эту зависимость и уравнения (1)-(3) рассчитывали величину излучательной способности $\varepsilon$ данного конкретного образца.

Далее, опять же без разгерметизации камеры, ее заполняли рабочим газом ( $\mathrm{Ar}$ или $\left.\mathrm{N}_{2}\right)$ до рабочего давления, и при закрытой заслонке 2 (рис. 2,a) включали и стабилизировали магнетронный разряд в течение 10-15 min, после чего открывали заслонку и проводили измерение временной зависимости температуры образца $T_{\text {samp }}$ уже в процессе роста пленки. По окончании измерения образец охлаждали до комнатной температуры и в том же вакуумном цикле по методике, описанной выше, вновь проводили измерение излучательной способности образца. Эта процедура делалась с целью повышения точности определения как излучательной способности, так и $T_{\text {surf. }}$

Отметим, что для исключения влияния возможного эффекта температурной зависимости $\varepsilon$ температуры, при которых измеряли $T_{\text {surf }}$ и $\varepsilon$, устанавливали максимально близкими. Определенная таким методом величина $\varepsilon$ в точности соответствовала излучательной способности поверхности образца в данном конкретном эксперименте, что позволяло рассчитывать температуру поверхности конденсации по уравнению (5) в процессе формирования на ней пленки с хорошей точностью.

Излучательная способность $\varepsilon$ пленок TiN, осажденных в различных условиях, находилась в пределах $0.12 \leq \varepsilon \leq 0.17$. Такой разброс $\varepsilon$ от эксперимента к эксперименту обусловлен различием в шероховатости поверхности образцов, каждый из которых готовился отдельно для каждой серии экспериментов. Погрешность измерения $T_{\text {surf }}$ лежала в пределах $\pm 3-5 \%$. Эта величина была получена на основании 3-5 измерений для каждой плотности потока энергии на поверхность, $q_{\text {in }}$, пропорциональной скорости роста пленки, которая, в свою очередь, пропорционально зависела от плотности разрядного ионного тока на мишень.

Экспериментальная простота разработанных нами методик измерения температуры и излучательной способности образца, а также прозрачность физических законов, лежащих в основе этих методов, дает уверенность в достоверности полученных результатов.

\section{2. Результаты}

Зависимости температур - образца, $T_{\text {samp }}$, измеренной термопарой, поверхности $T_{\text {surf, }}$ рассчитанной по уравнению (5), и их разности, $\Delta T=T_{\text {surf }}-T_{\text {samp }}$, как функции плотности потока энергии, $q_{\text {in }}$ (уравнение $\left.(1)\right)$, поступающей на поверхность конденсации в процессе осаждения пленок нитрида титана, полученных реакционным $\left(\mathrm{Ti}-\mathrm{in}-\mathrm{N}_{2}\right)$ и безреакционным $\left(\mathrm{TiN}-\mathrm{in}-\mathrm{N}_{2}\right)$ методами распыления, приведены на рис. 3, $a, b$. На рис. 3, представлена зависимость температуры поверхности конденсации $T_{\text {surf }}$ от плотности потока $q_{\text {in }}$ для разных случаев распыления. Сравнивая величины температуры поверхности, зафиксированной при равных на нее потоках энергии $q_{\text {in }}$, можно видеть, что максимальная температура была в случае реакционного распыления $\mathrm{Ti}-\mathrm{in}-\mathrm{N}_{2}$, а минимальная при распылении мишени из титана в аргоне - Ti-in-Ar (рис. $3, c)$.

Зависимости скорости роста пленок $\mathrm{TiN}, v_{g}$, от плотности потока энергии на поверхность конденсации, $q_{\text {in }}$, представлены на рис. 4. Эти данные были использованы 

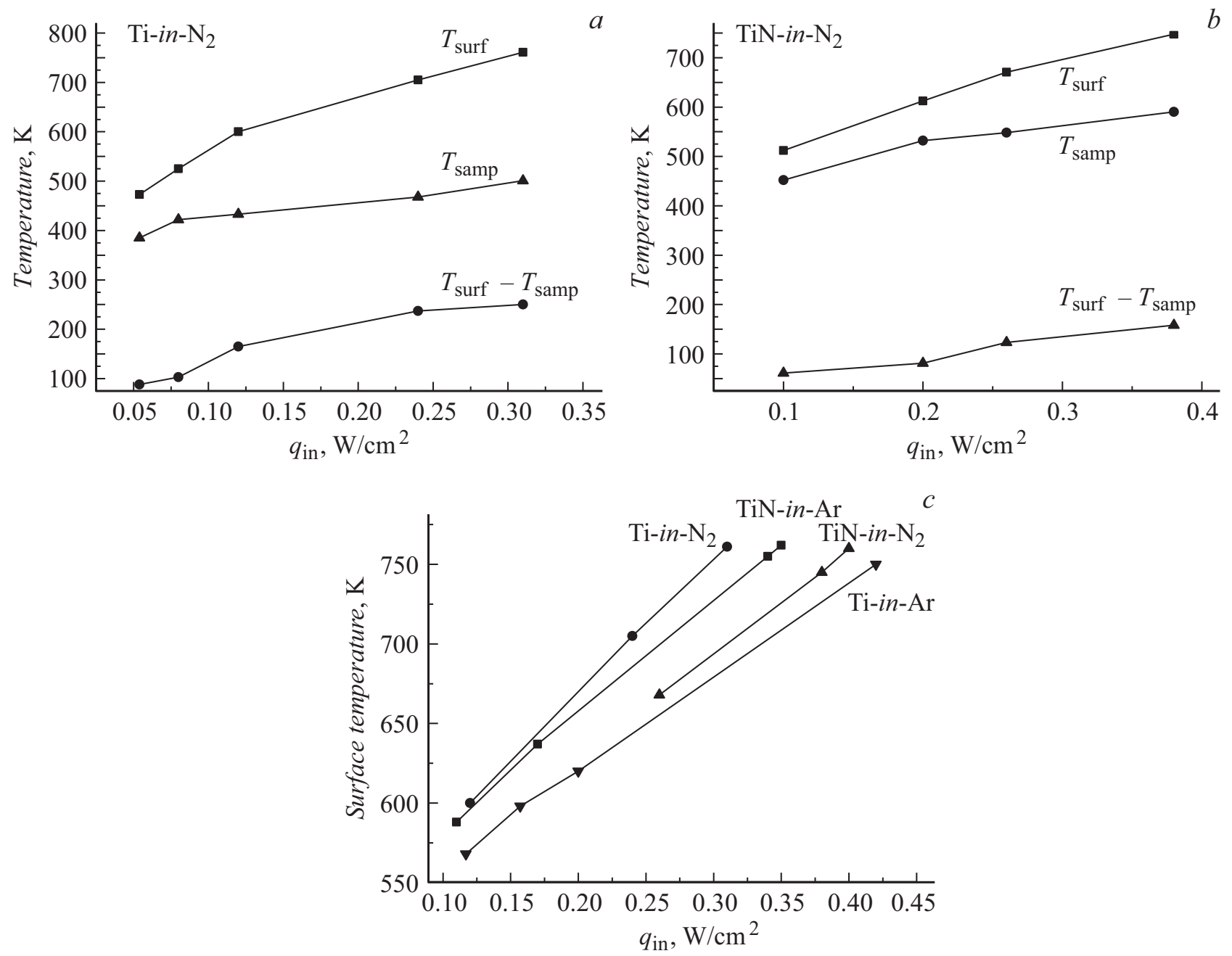

Pис. 3. Графики температуры: поверхности, $T_{\text {surf, }}$ образца, $T_{\text {samp }}$, и их разности, $\Delta T=\left(T_{\text {surf }}-T_{\text {samp }}\right)$ как функции потока энергии $q_{\text {in }}$, падающей на поверхность конденсации, для двух модификаций распыления: $a-\mathrm{Ti}-\mathrm{in}-\mathrm{N}_{2}\left(\right.$ реакционное); $b-\mathrm{TiN}-\mathrm{in}-\mathrm{N}_{2}$ (безреакционное); $c$ - рассчитанная на основании экспериментальных измерений температура поверхности, $T_{\text {surf }}$, как функция потока энергии $q_{\text {in }}$, на поверхность конденсации, для четырех модификаций распыления.

для расчета скорости потока пленкообразующих частиц, $f_{\text {at, }}$ поступающих на единицу площади поверхности конденсации.

Эта величина рассчитывалась, как

$$
f_{\text {at }}=n v_{g},
$$

где $n-$ число молекул TiN в $\mathrm{cm}^{3}$, рассчитанных, как $n=\rho / m_{\mathrm{TiN}}=5 \cdot 10^{22} \mathrm{~cm}^{-3}, \rho-$ плотность нитрида титана, $m_{\mathrm{TiN}}=\left(m_{\mathrm{Ti}}+m_{\mathrm{N}}\right)-$ молекулярный вес молекулы TiN в a.m.n., $v_{g}$ - скорость роста пленки.

Пленки, нанесенные во всех трех режимах на подложки из кремния и каменной соли, кристаллизовались в кубической решетке типа $\mathrm{NaCl}$ с параметром $a=4.24 \pm 0.02 \AA$, характерным для нитрида титана. Типичные для этих пленок электронограммы приведены на рис. 5. Как видно из электронограмм, все пленки текстурированы и имеют близкие ориентации плоскостей. Сравнение дифракционных линий на электронограммах от пленок, полученных в режимах (TiN-in-Ar) и $\left(\mathrm{Ti}-\mathrm{in}-\mathrm{N}_{2}\right)$ показывает, что размер областей когерентного рассеяния у пленок, в последнем режиме, несколько больше. Это следует из тех фактов, что ширина линий несколько меньше, а степень текстурированности несколько лучше в пленке, полученной в режиме $\mathrm{Ti}-\mathrm{in}-\mathrm{N}_{2}$. Исследование состава пленок и мишени показало, что отношение в них $\mathrm{N} / \mathrm{Ti} \approx 1$, т.е. соответствует стехиометрическому.

\section{3. Обсуждение результатов}

В исследованиях энергетических процессов, происходящих на поверхности конденсации во время магнетронного распыления разных металлов, было показано [11-13], что существуют различные источники энергии, поступающей на поверхность, ответственные за повышение температуры подложки в процессе роста пленки: (i) экзотермическое выделение теплоты 


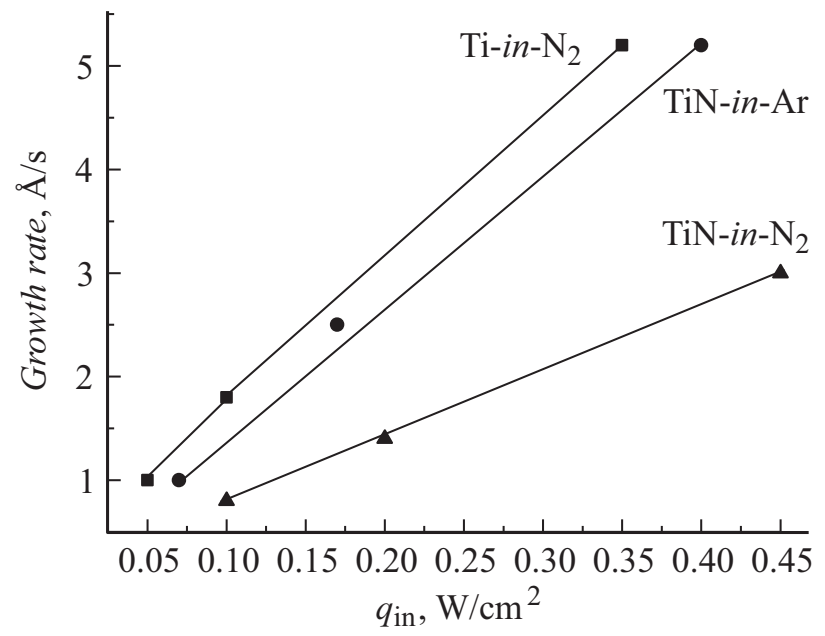

Рис. 4. Скорость роста пленок, осажденных в различных модификациях распыления, как функция потока энергии, $q_{\mathrm{in}}$, падающей на поверхность конденсации в процессе роста пленки.

при конденсации атомов (теплота конденсации), $E_{c}$; (ii) кинетическая энергия распыленных атомов, $E_{\mathrm{kin}}$; (iii) кинетическая энергия частиц (рекомбинированных атомов, молекул) рабочих газов, упруго отраженных от мишени и бомбардирующих поверхность конденсации, названной энергией из плазмы, $E_{\mathrm{pl}}$ [11]. Существует еще один тип энергии, который может влиять на температуру поверхности, это теплота химических реакций, выделяющаяся в различных процессах при нанесении пленок [14]. В работе [15] продемонстрировано успешное применение тепловой релаксационной калориметрии для исследования воздействия плазмы на поверхность подложки во время ее окисления и травления. Было показано, что повышение температуры подложки во время ее окисления (травления) обусловлено диссоциацией на ее поверхности молекул кислорода. В работе [16] авторы также наблюдали повышение температуры подложки вследствие химических реакций, происходящих на ее поверхности.

При получении пленок соединений любым из методов физического реакционного осаждения, в частности реакционным магнетронным распылением, также имеет смысл учитывать выделение теплоты экзотермических химических реакций, $E_{\text {chem }}$, происходящих на поверхности конденсации при формировании пленки соединения, как дополнительный источник энергии, повышающий температуру подложки. Очевидно, что вклад этой энергии может повлиять на температуру подложки только в случае, если реакция между компонентами при формировании пленок оксидов, нитридов, карбидов и т.д. происходит на поверхности конденсации, а не в газовой фазе между мишенью и подложкой.

Сумму энергий $q_{\text {in }}$ всех рассмотренных выше источников, выделяющихся в процессе роста пленки, запи- шем как

$$
q_{\text {in }}=f_{\text {at }}\left(E_{c}+E_{\text {kin }}+E_{\mathrm{pl}}+E_{\text {chem }}\right),
$$

где $f_{\text {at }}$ рассчитывается из уравнения (6), а другие члены уравнения (7) рассмотрены выше.

Предварим обсуждение полученных результатов следующими замечаниями: (i) члены $E_{\text {kin }}$ и $E_{\mathrm{pl}}$ в уравнении (7) являются функциями условий осаждения пленок: давления рабочего газа, расстояния „мишень-подложка“, типа магнетрона (сбалансированный или нет). Поскольку условия наших экспериментов близки к описанным в работе [11], величины $E_{\mathrm{kin}}$ и $E_{\mathrm{pl}}$ в расчетах мы возьмем из [11] как оценочные; (ii) условия в наших экспериментах были такими, в которых ППЧ, распыленные с мишени, преодолевали расстояние „мишень-подложка“ без взаимных столкновений и без столкновений с атомами рабочего газа.

Основываясь на результатах наших экспериментов и рассуждениях, приведенных выше, а также на данных, заимствованных из [11] и работы [17], посвященной детальному изучению термодинамических свойств нитрида титана (таблица), рассмотрим возможные механизмы формирования пленок TiN, осажденных в трех режимах магнетронного распыления, и энергетические эффекты каждого из механизмов.

Очевидно, что существует только три возможных комбинации типа пленкообразующих частиц, которые полностью определяют состав и структуру пленки TiN: (i) атомы Ti и N; (ii) молекулы TiN; (iii) комбинация атомов Ti, N и молекул TiN. Рассмотрим, какие возможны химические реакции и какая энергия может быть выделена на поверхности конденсации в каждом из этих случаев.

(i) На поверхность конденсации поступают только атомы Ti и N. В этом случае пленка TiN формируется на поверхности конденсации по реакции $\mathrm{Ti}+\mathrm{N}=\mathrm{TiN}_{\text {solid }}$. Общая энергия, выделяющаяся в этом случае, состоит из суммы $E_{c}(\mathrm{Ti})+E_{\text {kin }}(\mathrm{Ti})+E_{\mathrm{pl}}(\mathrm{Ti})+E_{\text {chem, }}$, где для атомов титана $E_{c}(\mathrm{Ti})=4.9 \mathrm{eV} \cdot \mathrm{at}^{-1}, \quad E_{\mathrm{kin}}(\mathrm{Ti}) \sim$ $\sim 8 \mathrm{eV} \cdot \mathrm{at}^{-1}, E_{\mathrm{pl}}(\mathrm{Ti}) \sim 9 \mathrm{eV} \cdot \mathrm{at}^{-1}$. Тогда как $E_{c}(\mathrm{Ti})$ имеет точное значение и не зависит от условий эксперимента, величины $E_{\mathrm{kin}}(\mathrm{Ti})$ и $E_{\mathrm{pl}}(\mathrm{Ti})$, взятые из $[11]$, используются нами как оценочные. Член $E_{\text {chem }}$ есть сумма энергий следующих химических процессов: $E_{\text {chem }}(\mathrm{I})=$ $=(\mathrm{Ti}+\mathrm{N})=4.8 \mathrm{eV} \cdot \mathrm{at}^{-1}-$ теплота, выделяющаяся при образовании молекулы TiN из отдельных атомов титана и азота, и $E_{\text {chem }}(\mathrm{II})=\mathrm{TiN}+\mathrm{TiN}=8.5 \mathrm{eV} \cdot \mathrm{at}^{-1}-$ теплота образования твердого нитрида титана из молекул TiN. Обе эти величины взяты из [17] (см. таблицу). Тогда суммарная энергия, выделяющаяся на поверхности конденсации при формировании пленки TiN из отдельных атомов титана и азота, составит

$$
\begin{aligned}
E_{0}(\mathrm{Ti}+\mathrm{N})= & E_{c}(\mathrm{Ti})+E_{\mathrm{kin}}(\mathrm{Ti})+E_{\mathrm{pl}}(\mathrm{Ti})+E_{\text {chem }}(\mathrm{I}) \\
& +E_{\text {chem }}(\mathrm{II}) \approx 35 \mathrm{eV} \cdot \mathrm{at}^{-1}
\end{aligned}
$$


$a$

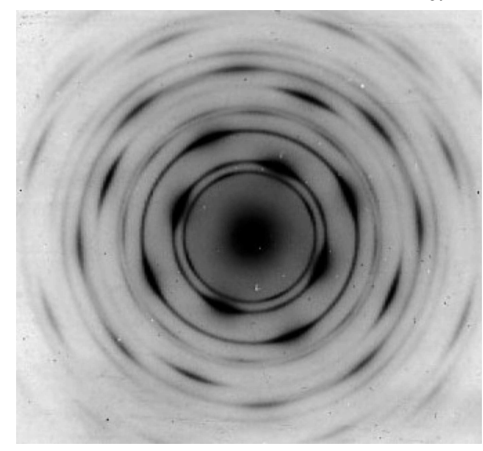

Ti- $-i n-\mathrm{N}_{2}$

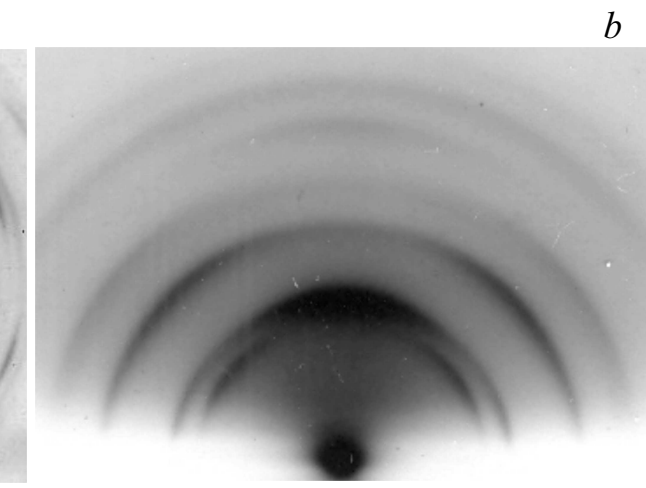

TiN-in- $\mathrm{N}_{2}$

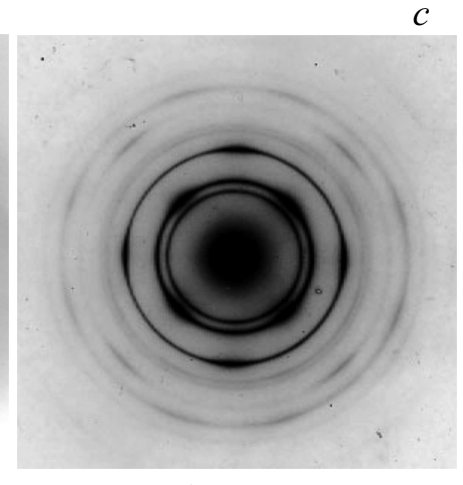

TiN-in-Ar

Рис. 5. Типичные электронограммы пленок TiN, осажденных на сколы монокристаллов $\mathrm{NaCl}(a, c)$ и на кремний $(b): a-$ $\mathrm{Ti}-\mathrm{in}-\mathrm{N}_{2}, b-\mathrm{TiN}-\mathrm{in}-\mathrm{N}_{2}, c-\mathrm{TiN}-\mathrm{in}-\mathrm{Ar}$.

Типы химических реакций, физические эффекты, сопровождающие эти реакции и энергии реакций

\begin{tabular}{|c|c|c|}
\hline Тип реакции & Физический эффект & Энергия эффекта $\left(\mathrm{eV} \cdot \mathrm{at}^{-1}\right)$ \\
\hline $\operatorname{TiN}(s) \leftrightarrow \operatorname{TiN}(g)\left(\Delta \mathrm{H}_{S, 298}^{0}\right)$ & $\begin{array}{c}\text { Образование твердого } \operatorname{TiN}(s) \text { из молекул }=E_{c} \\
\quad(\text { теплота конденсации молекулы TiN })\end{array}$ & 8.5 \\
\hline $\operatorname{TiN}(s) \leftrightarrow \operatorname{Ti}(g)+\mathrm{N}(g)\left(\Delta \mathrm{H}_{\mathrm{atm}, 298}^{0}\right)$ & Образование твердого $\mathrm{TiN}(\mathrm{s})$ из Тi и $\mathrm{N}$ атомов & 13.3 \\
\hline $\operatorname{Ti}(s) \leftrightarrow \operatorname{Ti}(g)\left(\Delta \mathrm{H}_{v, 298}^{0}\right)$ & Конденсация атома $\mathrm{Ti}=E_{c}($ теплоте конденсации атома $\mathrm{Ti})$ & 4.9 \\
\hline $\operatorname{TiN}(g) \leftrightarrow \operatorname{Ti}(g)+\mathrm{N}(g)\left(\mathrm{D}_{298}^{0}\right)$ & Образование молекулы TiN из Ti и $\mathrm{N}$ атомов & 4.8 \\
\hline
\end{tabular}

Примечание: Данные взяты из работы [17]. Для удобства величины энергии этих эффектов переведены из $\mathrm{kJ} \cdot \mathrm{mol}^{-1} \mathrm{~B}^{\mathrm{eV}} \cdot \mathrm{at}^{-1}$.

(ii) Пусть пленка формируется только из отдельных молекул TiN. В этом случае теплота образования твердого $\mathrm{TiN}(s)$ из молекул TiN будет равна теплоте конденсации молекулы нитрида титана, т.е. $E_{c}(\mathrm{TiN})=E_{\text {chem }}(\mathrm{II})=8.5 \mathrm{eV} \cdot \mathrm{at}^{-1}$. О кинетической энергии молекулы $\mathrm{TiN}$, распыленной из мишени, $E_{\text {kin }}(\mathrm{TiN})$, ничего неизвестно. Однако, ясно, что она будет меньше $E_{\text {kin }}(\mathrm{Ti})$ атома титана. В самом деле, при прочих равных условиях эксперимента (в частности, напряжении на мишени) кинетическая энергия, передаваемая ионами аргона атомам титана в случае мишени из титана, или молекулам TiN в случае мишени из нитрида титана, будет одной и той же. Но поскольку масса молекулы TiN больше массы атома Ti, то ясно, что $E_{\text {kin }}(\mathrm{TiN})<E_{\text {kin }}(\mathrm{Ti})$. Ввиду того, что относительно величины $E_{\mathrm{pl}}(\mathrm{TiN})$ также ничего неизвестно, то положим ее $E_{\mathrm{pl}}(\mathrm{TiN}) \sim E_{\mathrm{pl}}(\mathrm{Ti})$. С учетом рассуждений выше, находим

$$
E_{0}(\mathrm{TiN})=E_{c}(\mathrm{TiN})+E_{\mathrm{kin}}(\mathrm{TiN})+E_{\mathrm{pl}}(\mathrm{TiN}) \leq 26 \mathrm{eV} \cdot \mathrm{at}^{-1} .
$$

Это энергия, которая выделяется на поверхности конденсации при условии, что пленка нитрида титана формируется только из молекул TiN. А это означает, что тепло, выделяемое на поверхности конденсации в случае формирования пленки сугубо из атомов азота и титана больше, чем если пленка формируется из молекул $E_{0}(\mathrm{Ti}+\mathrm{N})>E_{0}(\mathrm{TiN})$. Следовательно, логично ожидать, что и температура поверхности $T_{\text {surf }}$ в первом случае будет выше.

(iii) Из рассмотрения этих двух случаев ясно, что для промежуточного случая, если поток пленкообразующих частиц будет состоять из атомов $\mathrm{Ti}, \mathrm{N}$ и молекул $\mathrm{TiN}$, то и энергия, выделяемая на поверхности конденсации в этом случае $-E_{0}(\mathrm{Ti}+\mathrm{N}, \mathrm{TiN})$, также будет иметь промежуочную величину.

(iv) И наконец, для случая распыления титана в аргоне, когда пленка формируется только из атомов титана, получаем

$$
E_{0}(\mathrm{Ti})=E_{c}(\mathrm{Ti})+E_{\mathrm{kin}}(\mathrm{Ti})+E_{\mathrm{pl}}(\mathrm{Ti}) \approx 22 \mathrm{eV} \cdot \mathrm{at}^{-1} .
$$

Сравнивая теперь результаты приведенных выше рассуждений и расчетов, получаем

$$
\begin{aligned}
E_{0}(\mathrm{Ti}+\mathrm{N}) & \approx 35 \mathrm{eV} \cdot \mathrm{at}^{-1}>E_{0}(\mathrm{TiN}) \approx 26 \mathrm{eV} \cdot \mathrm{at}^{-1} \\
& >E_{0}(\mathrm{Ti}) \approx 22 \mathrm{eV} \cdot \mathrm{at}^{-1} .
\end{aligned}
$$

В то же время температуры поверхности (для $\left.q_{\text {in }} \approx 0.3 \mathrm{~W} \cdot \mathrm{cm}^{-2}\right)$, измеренные для различных модификаций распыления, распределятся как

$$
\begin{aligned}
& T_{\text {surf }}\left(\mathrm{Ti}-\mathrm{in}-\mathrm{N}_{2}\right) \approx 760>T_{\text {surf }}(\mathrm{TiN}-\mathrm{in}-\mathrm{Ar}) \\
& \quad \approx 725>T_{\text {surf }}\left(\mathrm{TiN}-\mathrm{in}-\mathrm{N}_{2}\right) \approx 680>T_{\text {surf }}(\mathrm{Ti}-\mathrm{in}-\mathrm{Ar}) \\
& \quad \approx 660 \mathrm{~K}(\text { рис. } 3, c) .
\end{aligned}
$$


Величина $q_{\text {in }} \approx 0.3 \mathrm{~W} \cdot \mathrm{cm}^{-2}$ была выбрана как промежуточное значение во всем интервале потоков $q_{\text {in }}$, измеренных в эксперименте. Кроме того, выбор этого значения позволяет сравнить величины $T_{\text {surf }}$ для всех исследованных модификаций магнетронного распыления.

Корреляция между величинами оценочных значений потоков энергии для различных механизмов формирования пленок нитрида титана, $E_{0}$, и экспериментально измеренных значений $T_{\text {surf }}$ для разных модификаций распыления, позволяют предположить, что именно эти механизмы реализуются в реальности. А именно, формирование пленки $\mathrm{TiN}$ в случае $\left(\mathrm{Ti}-\mathrm{in}-\mathrm{N}_{2}\right)$ преимущественно осуществляется по реакции $\left(\mathrm{Ti}_{\mathrm{am}}+\mathrm{N}_{\mathrm{am}}\right)=\mathrm{TiN}_{\text {solid }}$, тогда как для безреакционных модификаций - (TiN-in-Ar) и $\left(\mathrm{TiN}-\mathrm{in}-\mathrm{N}_{2}\right)$ пленка преимущественно формируется из молекул TiN. Более высокая температура поверхности, измеренная для случая (TiN-in-Ar) $-T_{\text {surf }} \approx 725 \mathrm{~K}$, чем для случая ( $\left.\mathrm{TiN}-\mathrm{in}-\mathrm{N}_{2}\right)-T_{\text {surf }} \approx 680 \mathrm{~K}$, объясняется несколько большей величиной $E_{\mathrm{pl}}$ для ( $\left.\mathrm{TiN}-\mathrm{in}-\mathrm{Ar}\right)$. Это вполне резонно, если учесть существенную разницу в массах быстрых атомов аргона (40 a.m.u.) и нейтральных молекул азота $\mathrm{N}_{2}$ (28 a.m.u.), отраженных от мишени в сторону растущей пленки.

Еще одним косвенным свидетельством того, что $T_{\text {surf }}$ для $\left(\mathrm{Ti}-\mathrm{in}-\mathrm{N}_{2}\right)$ случая выше, чем для ( $\left.\mathrm{TiN}-\mathrm{in}-\mathrm{Ar}\right)$, является несколько меньший размер ОКР в этой пленке (рис. 5,c).

Стоит напомнить, что формирование пленки $\mathrm{TiN}$ по механизму $\left(\mathrm{Ti}_{a t}+\mathrm{N}_{\mathrm{am}}\right)=\mathrm{TiN}_{\text {solid }}$ подтверждается прямыми исследованиями состава ППЧ при реакционном распылении титана [7] и сплава (WTi) [18] в среде азота. В обоих случаях основными ППЧ, обнаруженными в газовой фазе, были атомы Ті и $\mathrm{N}$, наряду с которыми также присутствовало небольшое количество молекул TiN ( 1\% в [7] и $\sim 17 \%$ в [18]).

\section{Заключение}

Измерение температуры поверхности конденсации в процессе нанесения пленок нитрида титана различными модификациями магнетронного распыления, позволяет заключить, что:

1. Пленка, осажденная распылением мишени из титана в среде азота, формируется на поверхности конденсации преимущественно из отдельных атомов Ті и $\mathrm{N}$, тогда как молекулы TiN присутствуют в меньшинстве. Выделение дополнительного тепла химических реакций наряду с другими типами энергии, рассеиваемой на поверхности роста, обеспечивает максимальную температуру для этого механизма роста пленки.

2. При безреакционном распылении мишени из нитрида титана $\left(\mathrm{TiN}-\mathrm{in}-\mathrm{Ar}\right.$ и $\left.\mathrm{TiN}-\mathrm{in}-\mathrm{N}_{2}\right)$ в среде аргона пленка преимущественно формируется из молекул TiN. Пренебрежимо малый вклад в энергетический баланс химических реакций на поверхности роста приводит к более низкой ее температуре. Наиболее низкая температура, зафиксированная в случае распыления $\mathrm{TiN}-\mathrm{in}-\mathrm{N}_{2}$, связана с минимальным потоком энергии на поверхность роста для этой модификации.

3. Обнаружить эти механизмы формирования пленок $\mathrm{TiN}$ оказалось возможным благодаря измерению температуры поверхности конденсации относительно простым и доступным калориметрическим методом, который оказался удобным инструментом для проведения подобных исследований. С другой стороны, эти механизмы должны быть подтверждены прямыми исследованиями состава пленкообразующих частиц наряду с количественным подходом к проблеме эмиссии молекул или больших атомных кластеров в процессе ионного распыления твердых тел.

Авторы выражают признательность В.М. Верещаке (ИПМ НАН Украины, Киев) и В.Н. Новиченко (Научнотехнический центр НАН Украины, Киев) за помощь в проведении исследования состава объектов экспериментов. Отдельная благодарность профессору, докт. физ.мат. наук В.Р. Шагиняну (Институт ядерных исследований РАН, Санкт-Петербург) за плодотворное обсуждение материалов, представленных в рукописи.

\section{Список литературы}

[1] Ивановский Г.Ф., Петров В.И. Ионно-плазменная обработка материалов. М.: Радио и связь, 1986. 231 с.

[2] Smith D.L. Thin-Film Deposition. N.Y.: McGraw-Hill, 1995. $616 \mathrm{p}$.

[3] Orhing M. Materials Science of ThinFilms. 2nd ed. London: Academic Press, 2001. 794 p.

[4] Han J.G. J. Phys. D: Appl. Phys. 2009. Vol. 42. P. 0430010430017.

[5] Manova D., Gerlach J.W., Mändl S. Materials. 2010. Vol. 3. P. 4109-4141.

[6] Avelar-Batista J.C., Wilson A.D., Davison A., Matthews A., Fancey K.S. // J. Vac. Sci. Technol. A. 2003. Vol. 21. P. 1702 1707.

[7] Shaginyan L.R., Han J.G. // Thin Solid Films. 2004. Vol. 458. P. 186-190.

[8] Petrov I., Myers A., Greene J.E., Abelson J.R. // J. Vac. Sci. Technol. A. 1994. Vol. 12. P. 2846-2854.

[9] Heinrich R., Wucher A. // Nucl. Instrum. Meth. B. 1998. Vol. 140. P. 27-38.

[10] Shaginyan L.R., Kim Y.J., Britun N.V., Han J.G., Musil J., Belousov I.V. // Surf. Coat. Tech. 2007. Vol. 202. P. 486-493.

[11] Thornton J.A. // Thin Solid Films. 1978. Vol. 54. Vol. 23-31.

[12] Andritschky M., Guimardes F., Teixeira V. // Vacuum 1993. Vol. 44. P. 809-813.

[13] Kersten H., Deutsch H., Steffen H, Kroesen G.M.W., Hippler R. // Vacuum 2011. Vol. 63. P. 385-401.

[14] Hofer W.O. Sputtering by particle bombardment Ed. by R. Berisch, K. Wittmaack. Vol. III. Berlin: Springer. 1991. P. 67-81.

[15] Shivashankar S.A., Robinson B. // J. Vac. Sci. Technol. A. 1986. Vol. 4. P. $1826-1829$.

[16] Mozetič M., Zalar A., Drobnič M. // Thin Solid Films. 1999. Vol. 343-343. P. 101-104. 
[17] Steurns C.A., Kohl F.J. // The dissociation energy of gaseous titanium mononitride. NASA Technical NoteD5027. Lewis Research Center, Cleveland, Ohio. 1969. P. 1-20. https://ntrs.nasa.gov/archive/nasa/casi.ntrs.nasa.gov/ 19690009791.pdf

[18] Mišina M., Shaginyan L.R., Maček M., Panjan P. // Surf. Coat. Tech. 2001. Vol. 142-144. P. 348-354. 